Adhesive Bonding 


\section{Adhesive Bonding}

Edited by

Lieng-Huang Lee

Xerox Corporation

Webster, New York

Springer Science+Business Media, LLC 
Adhesive bonding, edited by Lieng-Huang Lee.

p. $c m$.

Includes bibliographical references and indexes.

1. Adhesives. I. Lee, Lieng-Huang, date.

TP968.A27 1991

$668^{\prime} .3--d c 20$

$90-15510$

\section{ISBN 978-1-4757-9008-5 ISBN 978-1-4757-9006-1 (eBook)}

DOI 10.1007/978-1-4757-9006-1

(C) 1991 Springer Science+Business Media New York

Originally published by Plenum Press, New York in 1991.

Softcover reprint of the hardcover 1st edition 1991

All rights reserved

No part of this book may be reproduced, stored in a retrieval system, or transmitted in any form or by any means, electronic, mechanical, photocopying, microfilming, recording, or otherwise, without written permission from the Publisher 


\section{Contributors}

E. H. Andrews - Department of Materials, Queen Mary and Westfield College, University of London, London E1 4NS, England

Sung Gun Chu - Hercules Incorporated, Research Center, Wilmington, Delaware 19894

H. M. Clearfield - IBM T. J. Watson Research Center, Yorktown Heights, New York 10598

Guy D. Davis - Martin Marietta Laboratories, Baltimore, Maryland 21227-3898

J. Thomas Dickinson - Department of Physics, Washington State University, Pullman, Washington 99164-2814

Jennifer A. Filbey - Finish Group, Hoechst Celanese, Charlotte, North Carolina 28232-2414.

Robert J. Good - Department of Chemical Engineering, State University of New York at Buffalo, Buffalo, New York 14260

Rakesh K. Gupta - Department of Chemical Engineering, State University of New York at Buffalo, Buffalo, New York 14260

Irvin M. Krieger - Center for Adhesives, Sealants, and Coatings, Case Western Reserve University, Cleveland, Ohio 44106

Raymond B. Krieger, Jr. - Engineering Materials Department, American Cyanamid Company, Havre de Grace, Maryland 21075

Lieng-Huang Lee - Webster Research Center, Xerox Corporation, Webster, New York 14580

Daniel Maugis - Laboratoire des Materiaux et Structures (UMR 113), CNRS-LCPC, 75732 Paris Cédex 15, France

D. K. McNamara - Martin Marietta Laboratories, Baltimore, Maryland 21227-3898

J. Dean Minford - Consultant, Hilton Head Island, South Carolina 29918

J. N. Reddy - The Center for Adhesive and Sealant Science and Department of Engineering Science and Mechanics, Virginia Polytechnic Institute and State University, Blacksburg, Virginia 24061

Joseph L. Rose - Department of Mechanical Engineering, Drexel University, Philadelphia, Pennsylvania 19104 
S. Roy - Engineering Materials Division, Southwest Research Institute, San Antonio, Texas 78228-0510

James P. Wightman - Center for Adhesive and Sealant Science and Chemistry Department, Virginia Polytechnic Institute and State University, Blacksburg, Virginia 24061 


\section{Preface}

For several years, I have been responsible for organizing and teaching in the fall a short course on "Fundamentals of Adhesion: Theory, Practice, and Applications" at the State University of New York at New Paltz. Every spring I would try to assemble the most pertinent subjects and line up several capable lecturers for the course. However, there has always been one thing missing - an authoritative book that covers most aspects of adhesion and adhesive bonding. Such a book would be used by the participants as a main reference throughout the course and kept as a sourcebook after the course had been completed. On the other hand, this book could not be one of those "All you want to know about" volumes, simply because adhesion is an interdisciplinary and ever-growing field.

For the same reason, it would be very difficult for a single individual, especially me, to undertake the task of writing such a book. Thus, I relied on the principle that one leaves the truly monumental jobs to experts, and I finally succeeded in asking several leading scientists in the field of adhesion to write separate chapters for this collection. Some chapters emphasize theoretical concepts and others experimental techniques. In the humble beginning, we planned to include only twelve chapters. However, we soon realized that such a plan would leave too much ground uncovered, and we resolved to increase the coverage. After the book had evolved into thirty chapters, we started to feel that perhaps our mission had been accomplished. Originally we had thought to publish the entire project under the title Fundamentals of Adhesion; then it was deemed necessary to split a portion of it off into this, the second volume, Adhesive Bonding. Since these two volumes include chapters by many authors, some overlap is inevitable.

In the first volume, we focused on adhesion with or without the use of an adhesive, and we were chiefly concerned with mechanisms of bond formation. In this volume, we are interested in both bond-forming and bond-breaking processes. Since polymeric adhesives will be at the center of our discussions, we need to know their physical properties as well as their related measurements. After we apply an adhesive to an adherend, we must understand how to determine bond strengths. With data on hand, we should also learn how to analyze the test results and make certain predictions about the durability of adhesive bonds. Though the entire process sounds rather difficult, we hope that readers will find this volume of benefit.

Thus in this volume we shall concentrate on the fundamentals of bonding with the aid of adhesives. Chapter 1 reviews the mechanisms of polymer adhesion and summarizes significant findings from the first volume. This introduction should serve as a bridge between the two volumes. Chapters 2 and 3 introduce rheological aspects of adhesives and adhesion. They are followed by Chapters 4 and 5, discussing measurements of rheological and dynamic properties of adhesives and sealants. Chapters 6 and 7 focus our attention on adherends by showing us new methods of characterizing them and related interfaces. Then Chapter 8 delineates various methods of surface preparation for adherends. Chapter 9 reviews various ways to prepare and determine durable adhesive joints. Chapter 10 serves to introduce new methods in measuring 
structural adhesive bonds and durability. The following chapters are more theoretical. Chapters 11 and 12 describe somewhat different views on the application of fracture mechanics to adhesive bonding. Chapter 13 is intended to treat some basic concepts about finite-element analysis and its applications to adhesive joints. Chapter 14 illustrates the physics of fracture in terms of fracto-emission. Finally, the last chapter leads us to applications of nondestructive testing (NDT) to adhesive joints. NDT is one of the important test methods for future adhesive and composite technology.

A book of this format can still fail in not covering the subject broadly and deeply enough. Regarding the breadth of the book, we deliberately omitted most of the organic chemistry and formulations of adhesives and sealants because there are other available books dealing with those subjects; two related books edited by me have been published in the last several years: Adhesive Chemistry: Developments and Trends (Plenum Press, 1984) and Adhesives, Sealants, and Coatings for Space and Harsh Environments (Plenum Press, 1988). Regarding the depth of the book, one may still object that none of the subjects has been treated sufficiently thoroughly. Yet, since this is only an introductory text, it is not our intention to discuss each subject at length. In fact, each chapter could have readily been expanded into a separate book, had that been the goal.

For better readability, all of the chapters have been refereed and separate nomenclature lists are included at the end of most of the chapters. I should like to take this opportunity to thank the many referees for their efforts. I also wish to thank all of the authors for their fine cooperation and patience. Finally, I sincerely appreciate the support of the Webster Research Center of Xerox Corporation in helping me to complete this task, and the assistance of Mr. F. G. Belli and Ms. E. Jonas of the Technical Information Center in preparing the indexes.

Lieng-Huang Lee 


\section{Contents}

\section{Recent Studies in Polymer Adhesion Mechanisms}

\section{Lieng-Huang Lee}

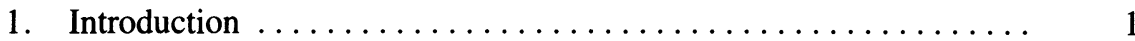

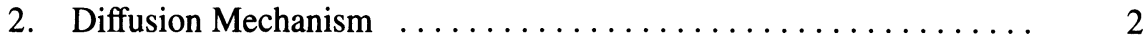

2.1. Viscoelastic Properties of Polymers ................. 2

2.2. Theories of Self-Diffusion $\ldots \ldots \ldots \ldots \ldots \ldots \ldots \ldots, 2$

2.3. Interdiffusion, Healing, and Welding $\ldots \ldots \ldots \ldots \ldots \ldots \ldots$

2.4. Tack and Green Strength $\ldots \ldots \ldots \ldots \ldots \ldots \ldots \ldots \ldots \ldots \ldots$

2.5. Fractals at an Interface $\ldots \ldots \ldots \ldots \ldots \ldots \ldots \ldots \ldots \ldots \ldots$

3. Lifshitz-van der Waals Interactions $\ldots \ldots \ldots \ldots \ldots \ldots \ldots \ldots \ldots$

3.1. Partial Wetting of Liquid $\left(\theta_{e}>0\right) \ldots \ldots \ldots \ldots \ldots \ldots . \quad 9$

3.2. Complete Wetting of Liquid $\left(\theta_{e} \rightarrow 0\right) \ldots \ldots \ldots \ldots \ldots \ldots \ldots \ldots \ldots \ldots$

3.3. Complete Wetting of Polymer Melt ................. 14

3.4. Dry Spreading of Polymer Solution ................. 14

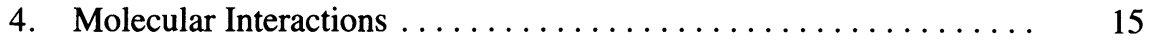

4.1. Enthalpy of the Acid-Base Interaction .............. 15

4.2. Work of Adhesion (The Acid-Base Component) ......... 15

4.3. Total Work of Adhesion ........................ 16

4.4. Extension of the Hard-Soft Acid-Base (HSAB)

Principle to Solid Adhesion .................... 16

5. Adsorption Mechanism-A Polymer at an Interface ........... 17

5.1. Adsorption Profile ........................... 17

5.2. Loops, Trains, and Tails .................... 18

5.3. Surface Energetics of Adsorption .................. 18

5.4. Mean Field versus Scaling Theories $\ldots \ldots \ldots \ldots \ldots \ldots . . . \ldots$

5.5. Role of Adsorption in Adhesion ................... 19

6. Mechanical Interlocking Mechanism $\ldots \ldots \ldots \ldots \ldots \ldots \ldots . \ldots . \ldots . \ldots$

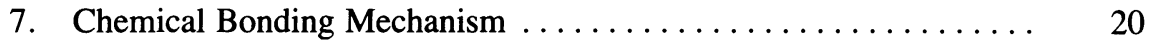

8. Electrostatic Mechanism ....................... 20

8.1. Direct Evidence of the Electrical Double Layer .......... 20

8.2. Work of Electrical Double Layer versus Peel Work ........ 21

8.3. Elimination of the Electrical Double Layer by Surface Modification .................... 21

8.4. Electrostatic Adhesion Between Anion and Cation Pairs ..... 21

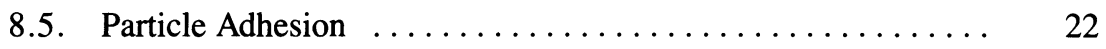




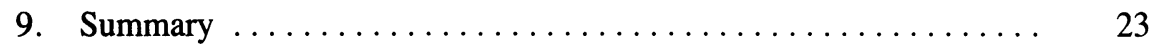

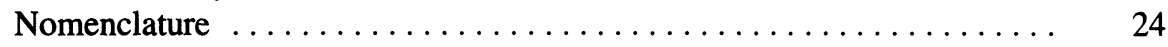

References $\ldots \ldots \ldots \ldots \ldots \ldots \ldots \ldots \ldots \ldots \ldots \ldots \ldots \ldots \ldots \ldots \ldots \ldots, 26$

\section{Flow Properties of Adhesives}

Irvin M. Krieger

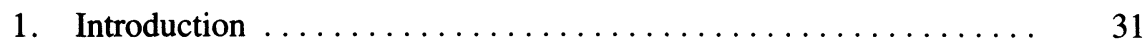

2. Rheometry $\ldots \ldots \ldots \ldots \ldots \ldots \ldots \ldots \ldots \ldots \ldots \ldots \ldots \ldots \ldots \ldots, \quad 32$

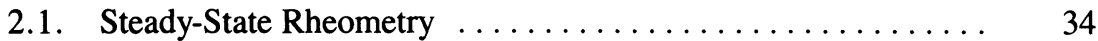

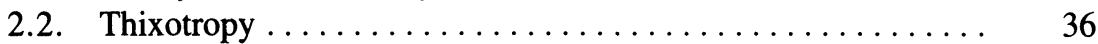

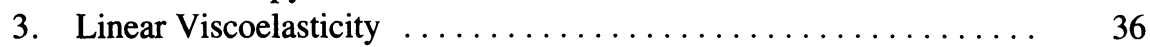

3.1. Oscillatory Measurements $\ldots \ldots \ldots \ldots \ldots \ldots \ldots \ldots \ldots \ldots \ldots$

3.2. Transient Analysis .......................... 37

3.3. Comparison of Transient and Dynamic Moduli ......... 38

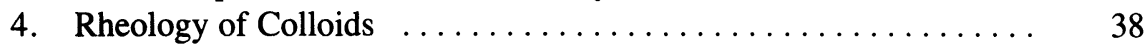

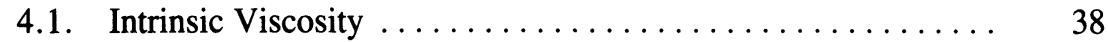

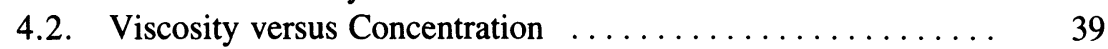

4.3. Non-Newtonian Effects . . . . . . . . . . . . . . . . . . $\quad 40$

4.4. Dimensional Analysis $\ldots \ldots \ldots \ldots \ldots \ldots \ldots \ldots \ldots \ldots, 41$

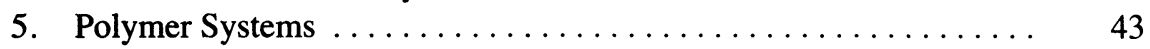

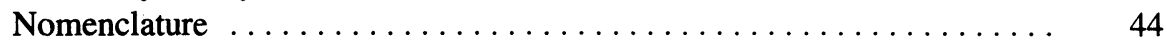

References $\ldots \ldots \ldots \ldots \ldots \ldots \ldots \ldots \ldots \ldots \ldots \ldots \ldots \ldots \ldots, 45$

\section{The Coupling of Interfacial, Rheological, and Thermal} Control Mechanisms in Polymer Adhesion

Robert J. Good and Rakesh K. Gupta

1. Introduction $\ldots \ldots \ldots \ldots \ldots \ldots \ldots \ldots \ldots \ldots \ldots \ldots \ldots \ldots \ldots, 47$

2. General Model for Separation of Thermoplastic

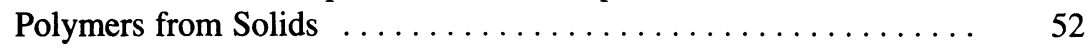

2.1. Elongational Work $\ldots \ldots \ldots \ldots \ldots \ldots \ldots \ldots \ldots \ldots \ldots \ldots$

2.2. Surface Work in a Real Separation Process ............ 54

2.3. The Processes as Alternatives $\ldots \ldots \ldots \ldots \ldots \ldots \ldots \ldots . \ldots \ldots$

3. Application of the Isothermal Theory $\ldots \ldots \ldots \ldots \ldots \ldots \ldots .61$

4. Thermal Control Mechanisms in Adhesion: Adiabatic Theory ..... 64

4.1. Pressure-Sensitive Adhesives .................... 64

4.2. Fracture of Polymers with High $T_{\mathrm{g}}$ Values $\ldots \ldots \ldots \ldots \ldots .65$

5. Application to Adhesion of High- $T_{\mathrm{g}}$ Polymers to Solids $\ldots \ldots \ldots$. 66

6. Discussion and Summary $\ldots \ldots \ldots \ldots \ldots \ldots \ldots \ldots \ldots \ldots \ldots \ldots \ldots \ldots$

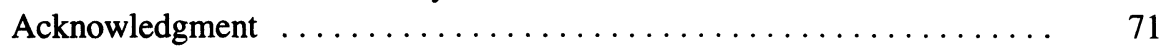

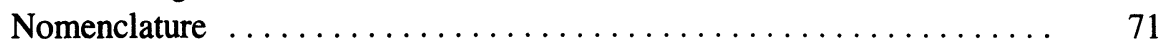

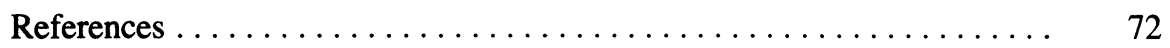




\section{Extensional Rheometry of Polymer Melts}

Rakesh K. Gupta

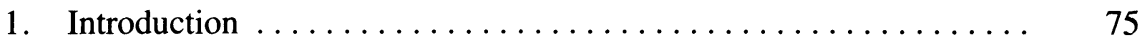

2. Kinematics of Extensional Flows $\ldots \ldots \ldots \ldots \ldots \ldots \ldots \ldots \ldots \ldots . \ldots \ldots$

3. The Stress Tensor in Uniaxial Extension $\ldots \ldots \ldots \ldots \ldots \ldots \ldots .78$

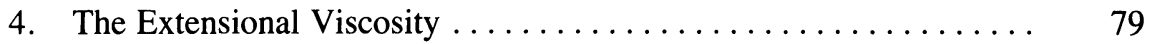

5. Uniform Stretching of Polymer Melts ................ 80

5.1. Sample Length Variable During the Test,

Constant Stretch Rate ....................... 81

5.2. Sample Length Variable During the Test, Constant Stress .... 82

5.3. Constant Sample Length Experiments ............... 83

5.4. Experimental Results $\ldots \ldots \ldots \ldots \ldots \ldots \ldots \ldots \ldots \ldots \ldots$

6. Nonuniform Stretching of Polymer Melts $\ldots \ldots \ldots \ldots \ldots \ldots \ldots . \quad 86$

6.1. Melt Spinning of Fibers $\ldots \ldots \ldots \ldots \ldots \ldots \ldots \ldots \ldots \ldots$

6.2. Converging Flow into a Capillary $\ldots \ldots \ldots \ldots \ldots \ldots \ldots . \quad 87$

6.3. Other Methods of Stretching Polymer Melts .......... 88

7. Constitutive Equations $\ldots \ldots \ldots \ldots \ldots \ldots \ldots \ldots \ldots \ldots \ldots \ldots \ldots \ldots \ldots \ldots \ldots$

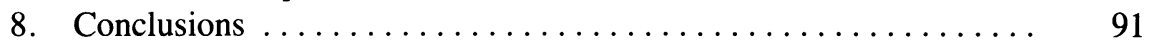

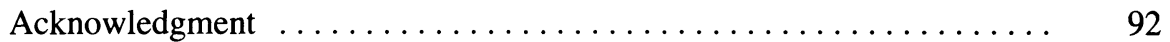

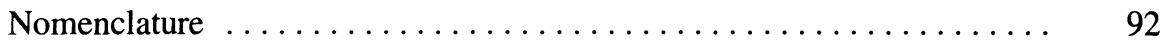

References ................................. 93

5. Dynamic Mechanical Properties of Pressure-Sensitive Adhesives

Sung Gun Chu

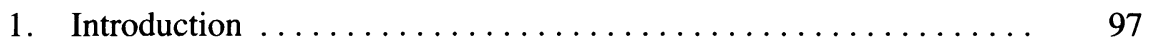

2. Dynamic Mechanical Testing $\ldots \ldots \ldots \ldots \ldots \ldots \ldots \ldots \ldots . \ldots . \ldots . \ldots 2$

3. Dynamic Mechanical Properties of Elastomers ............ 102

3.1. Natural Rubber ........................... 103

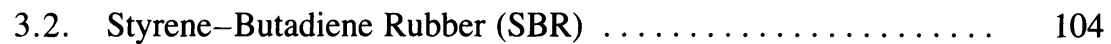

3.3. Block Copolymers ......................... 106

4. Dynamic Mechanical Properties of Tackifying Resins ........ 108

5. Dynamic Mechanical Properties of Commercial PSA ......... 108

6. Elastomer-Resin Blends ........................ 115

6.1. Natural Rubber and SBR $\ldots \ldots \ldots \ldots \ldots \ldots \ldots \ldots \ldots \ldots$

6.2. Block Copolymers ....................... 117

7. Pressure-Sensitive Adhesive Testing Methods $\ldots \ldots \ldots \ldots \ldots \ldots .122$

8. Relation Between the Dynamic Mechanical Properties and PSA Performance ........................... 124

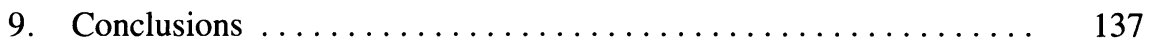

Acknowledgments ............................... 137

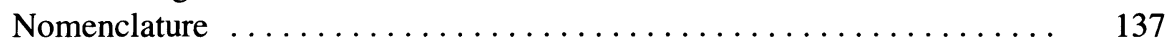

References .................................. 137 


\section{Characterization of Surfaces}

Guy D. Davis

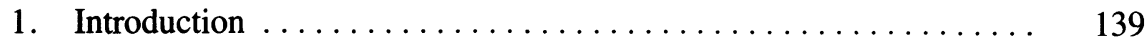

2. Surface-Sensitive Techniques $\ldots \ldots \ldots \ldots \ldots \ldots \ldots \ldots \ldots \ldots \ldots, 141$

2.1. X-ray Photoelectron Spectroscopy ................ 141

2.2. Auger Electron Spectroscopy/Scanning Auger Microscopy ... 144

2.3. Comparison of Techniques ...................... 148

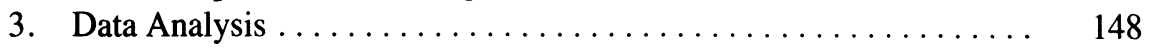

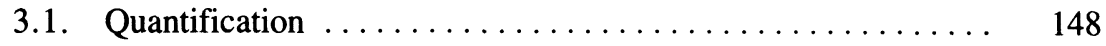

3.2. Chemical-State Information $\ldots \ldots \ldots \ldots \ldots \ldots \ldots \ldots \ldots, 153$

3.3. Depth-Distribution Information $\ldots \ldots \ldots \ldots \ldots \ldots \ldots \ldots$

3.4. Surface-Behavior Diagrams $\ldots \ldots \ldots \ldots \ldots \ldots \ldots \ldots . \ldots 1$

4. Applications to Adhesive Bonding $\ldots \ldots \ldots \ldots \ldots \ldots \ldots \ldots . \ldots 2$

4.1. Failure Analysis . ......................... 162

4.2. Hydration of Phosphoric-Acid-Anodized Aluminum ...... 165

4.3. Adsorption of Hydration Inhibitors . . . . . . . . . . . . 167

5. Conclusions ................................. 169

Acknowledgments . . . . . . . . . . . . . . . . . . . . . . . . 169

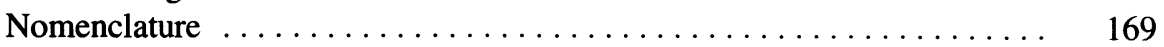

References ................................. 170

\section{Surface Characterization in Polymer/Metal Adhesion}

Jennifer A. Filbey and James P. Wightman

1. Introduction ................................ 175

2. Scanning Electron Microscopy (SEM) $\ldots \ldots \ldots \ldots \ldots \ldots \ldots .176$

3. Scanning Transmission Electron Microscopy (STEM) ........ 184

4. Surface Reflectance Infrared Spectroscopy (SRIRS) .......... 185

5. Inelastic Electron Tunneling Spectroscopy (IETS) _.......... 190

6. Auger Electron Spectroscopy (AES) $\ldots \ldots \ldots \ldots \ldots \ldots \ldots \ldots, 190$

7. Ion Scattering Spectroscopy (ISS) $\ldots \ldots \ldots \ldots \ldots \ldots \ldots \ldots \ldots \ldots$

8. Secondary Ion Mass Spectroscopy (SIMS) $\ldots \ldots \ldots \ldots \ldots \ldots \ldots$

9. X-ray Photoelectron Spectroscopy (XPS) $\ldots \ldots \ldots \ldots \ldots \ldots \ldots . . \ldots \ldots$

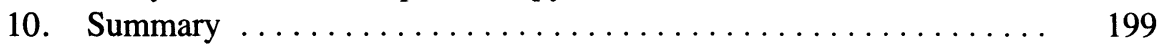

Acknowledgments ............................... 200

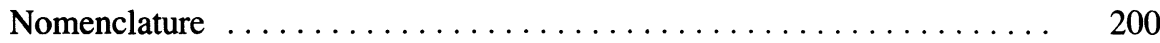

References $\ldots \ldots \ldots \ldots \ldots \ldots \ldots \ldots \ldots \ldots \ldots \ldots \ldots \ldots \ldots \ldots \ldots \ldots, 201$

\section{Adherend Surface Preparation for} Structural Adhesive Bonding

H. M. Clearfield, D. K. McNamara, and Guy D. Davis

1. Introduction ................................. 203

1.1. High-Resolution Scanning Electron Microscopy ......... 203

1.2. Surface Analysis . . . . . . . . . . . . . . . . . . . . . 205 
2. Aluminum Adherends . . . . . . . . . . . . . . . . . . . . . 205

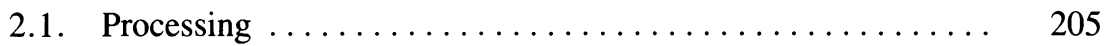

2.2. Oxide Morphology and Chemistry ............. 206

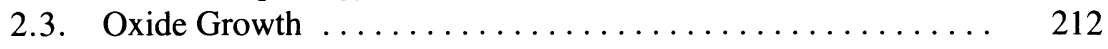

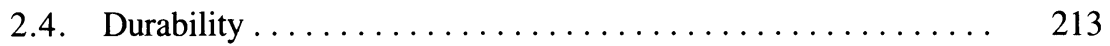

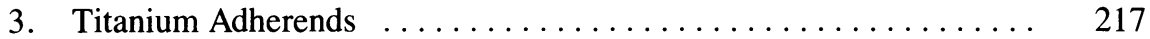

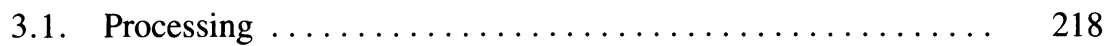

3.2. Oxide Morphology and Chemistry .............. 219

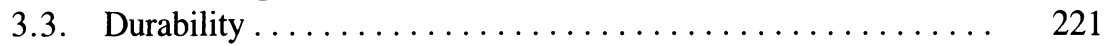

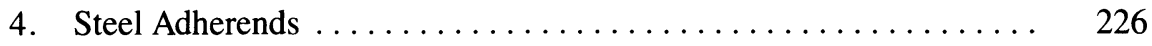

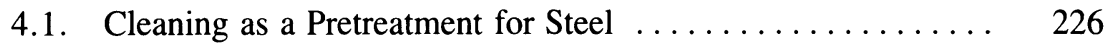

4.2. Chemical Etches ...................... 227

4.3. Conversion Coating Treatments $\ldots \ldots \ldots \ldots \ldots \ldots \ldots \ldots 231$

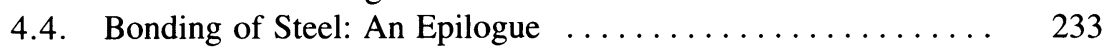

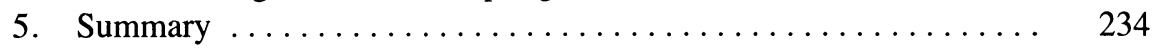

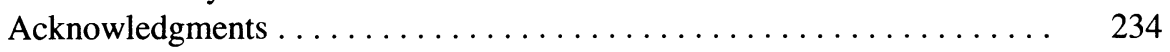

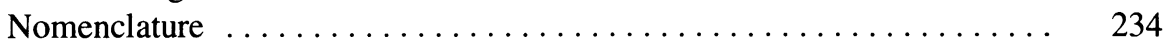

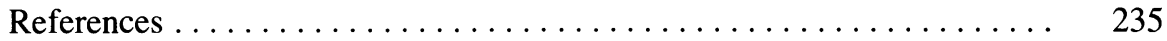

\section{Durability Evaluation of Adhesive Bonded Structures}

\section{J. Dean Minford}

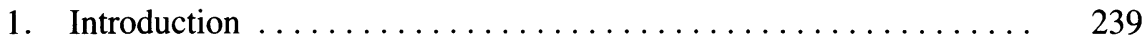

2. Chemical and Physical Attributes of Adhesion and Durability .... 240

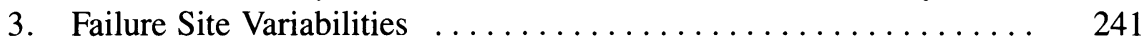

4. Bondline Characteristics Affecting Bond Performance ......... 242

4.1. Modulus of Elasticity and Coefficient

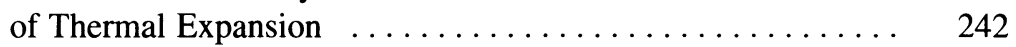

4.2. Interfacial Imperfections $\ldots \ldots \ldots \ldots \ldots \ldots \ldots \ldots \ldots \ldots \ldots \ldots \ldots \ldots \ldots$

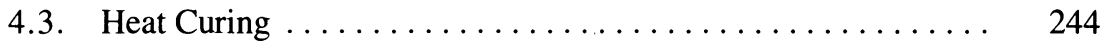

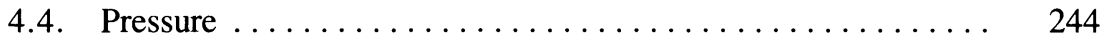

4.5. Mechanical Energy ..................... 245

4.6. Cohesive versus Adhesive Failure Observations ......... 245

5. Characteristics of Metal Adherend Surfaces ... . . . . . . . . . . 248

6. Effect of Water on Adhesive-Bonded Structures ............ 251

7. Bond-Joint Durability as a Function of Surface Pretreatment . . . . . 254

7.1. General Relationships ...................... 254

7.2. Effect of Specific Surface Pretreatments ............. 254

8. Test Environments for Determining the Durability

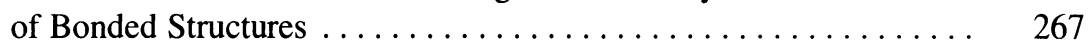

8.1. Static Heat Aging or Cryogenic Exposures . . . . . . . . . . 269

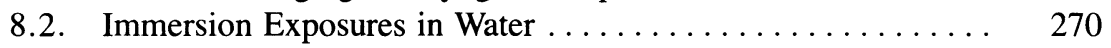

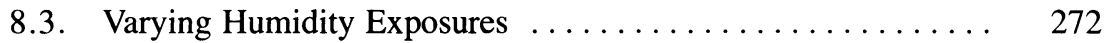

8.4. Wet, Freeze, and Thaw Cyclic Exposures ........... 272

8.5. Continuous or Intermittent Saltwater Immersion or Spray Exposure . . . . . . . . . . . . . . . 
8.6. Natural Atmospheric Exposures . . . . . . . . . . . . 275

8.7. Simultaneous Stress and Environmental Exposures ....... 276

8.8. Crack Initiation and Propagation with

Environmental Exposures

9. Durability of Structural Joints Assembled with

Combinations of Joining Procedures $\ldots \ldots \ldots \ldots \ldots \ldots \ldots \ldots 281$

10. Durability of Dissimilar Materials Structures ............. 282

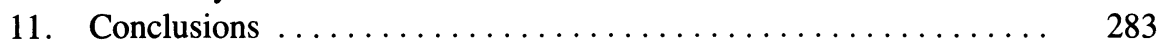

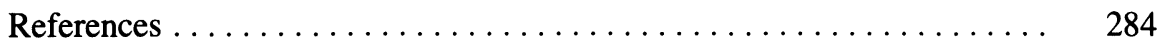

10. Testing Structural Adhesives for Properties Necessary for Stress Analysis

Raymond B. Krieger, Jr.

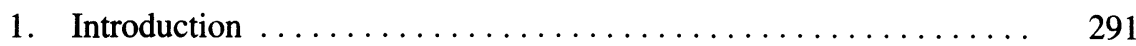

2. Early Tests for Adhesive Strength $\ldots \ldots \ldots \ldots \ldots \ldots \ldots \ldots \ldots . \ldots 291$

3. A Fundamental Stress Analysis for Bonded Metal Structure ..... 292

4. KGR-1, An Extensometer for Measuring Adhesive

Shear Strain versus Stress . . . . . . . . . . . . . . . . . . . . . . . . . 294

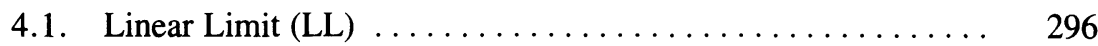

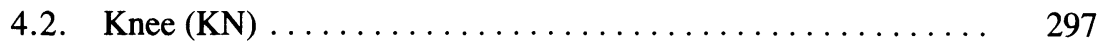

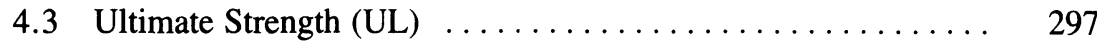

5. KGR-2, An Extensometer for Measuring Adhesive

Shear Strain in Bonded Structure . . . . . . . . . . . . . . . . . 297

6. The Relationship Between Adhesive Shear Strain Properties and Adhesive Performance in an Actual Structure . . . . . . . . 298

7. A Specimen Configuration for Testing Adhesives in Fatigue and Creep ......................... 301

References ............................. 302

\section{Adherence and Fracture Mechanics}

\section{Daniel Maugis}

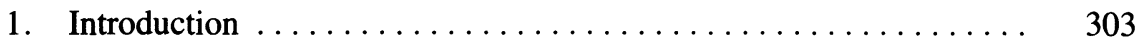

2. Adherence of Two Elastic Solids in Contact ............ 303

2.1. The Griffith Criterion for Equilibrium Contacts ... . . . . . 304

2.2. Stability of Equilibrium and Adherence Force .......... 306

2.3. The Griffith, Barenblatt, and Dugdale Models of Crack .... 307

2.4. Adherence of Punches as an Example ............... 309

2.5. Influence of Prestresses ................... 314

3. Adherence due to Liquid Bridges ................. 315

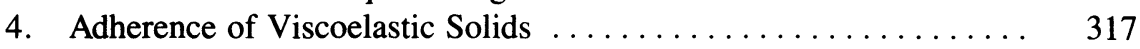

4.1. Tackiness ......................... 319

4.2. Viscoelastic Losses and Negative Resistance Branch ...... 319 
4.3. Velocity Jumps and Stick-Slip .............. 320

5. Viscous Drag and Limited Rate of Transport ............ 324

6. Dwell-Time Effects ........................ 325

7. The Problem of the Threshold Value $G_{0} \ldots \ldots \ldots \ldots \ldots \ldots . \ldots 327$

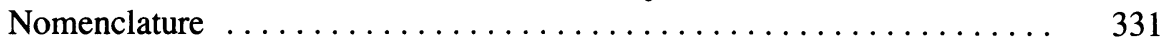

References .............................. 332

\section{Generalized Fracture Mechanics Approach to Adhesion}

E. H. Andrews

1. Introduction ................................ 337

2. Fracture Mechanics ......................... 337

3. Nonlinear, Finite-Strain Fracture Mechanics . . . . . . . . . . . . 339

4. Shortcomings of Conventional Fracture Mechanics ........... 341

5. Generalized Fracture Mechanics . . . . . . . . . . . . . . . 342

6. Putting Generalized Fracture Mechanics to Work ........... 344

7. Adhesion of Crosslinked Elastomers ... . . . . . . . . . . . . . . 345

8. Structural Adhesives ......................... 348

9. Moisture Attack on Interfaces . . . . . . . . . . . . . . . . . . . 349

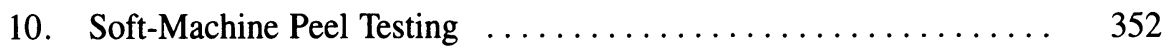

11. Conclusion .............................. 355

Nomenclature .............................. 356

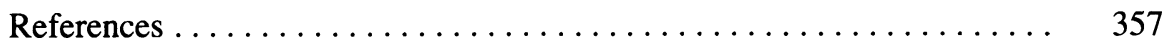

\section{Finite-Element Analysis of Adhesive Joints}

J. N. Reddy and S. Roy

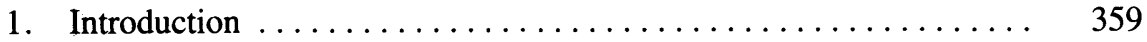

1.1. General Comments ...................... 359

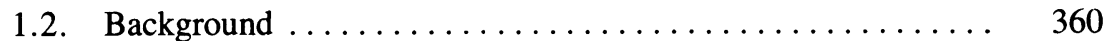

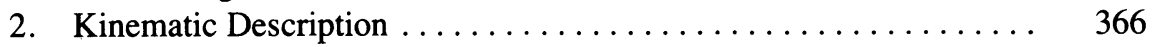

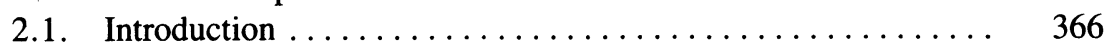

2.2. Incremental Equations of Motion $\ldots \ldots \ldots \ldots \ldots \ldots \ldots \ldots$

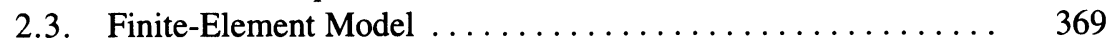

3. Viscoelastic Formulation ....................... 370

3.1. Introduction . . . . . . . . . . . . . . . . . . . . . . 370

3.2. One-Dimensional Model . . . . . . . . . . . . . . . . . . . 370

3.3. Two-Dimensional Formulation .................. 373

3.4. Finite-Element Model . . . . . . . . . . . . . . . . . . . . . 374

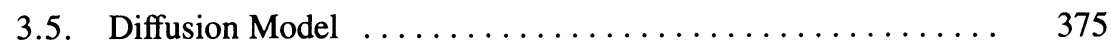

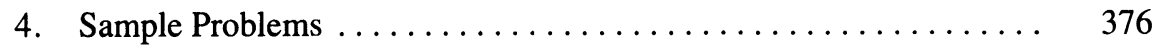

4.1. Preliminary Comments ...................... 376

4.2. Geometric Nonlinear Analysis . . . . . . . . . . . . . . . 377

4.3. Linear Viscoelastic Analysis .................... 377

4.4. Analysis of a Thick Adherend Specimen ........... 379 
4.5. Nonlinear Viscoelastic Analysis . . . . . . . . . .... 381

4.6. Nonlinear Viscoelastic Analysis of a Model Joint ........ 385

4.7. Analysis of a Single Lap Laminated Composite Joint ..... 385

4.8. Moisture Diffusion in a Butt Joint .............. 386

4.9. Analysis of a Bonded Cantilever Beam ............ 388

5. Summary ............................. 390

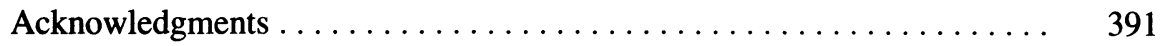

Nomenclature . . . . . . . . . . . . . . . . . . . . . . . . 391

References ................................ 392

\section{Fracto-Emission from Adhesive Failure}

\section{J. Thomas Dickinson}

1. Introduction . . . . . . . . . . . . . . . . . . . . . 395

2. Experimental ........................... 397

3. Results and Discussion $\ldots \ldots \ldots \ldots \ldots \ldots \ldots \ldots \ldots \ldots \ldots . \ldots \ldots$

3.1. Filled versus Unfilled Epoxy .................... 398

3.2. Chaotic/Fractal Nature of phE and Fracture

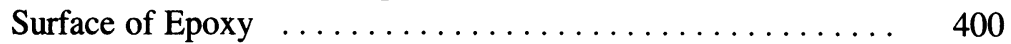

3.3. NE from Epoxy ......................... 404

3.4. phE from Embedded Interfaces $\ldots \ldots \ldots \ldots \ldots \ldots \ldots \ldots . . \ldots 408$

3.5. phE from Peeling Pressure-Sensitive Adhesives ......... 411

4. Conclusions ............................... 419

Acknowledgments . . . . . . . . . . . . . . . . . . . . . . . . 419

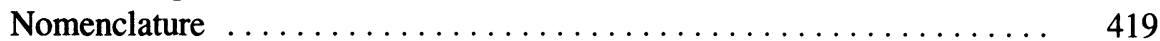

References ................................ 420

15. Ultrasonic Nondestructive Evaluation Technology for Adhesive Bond and Composite Material Inspection

Joseph L. Rose

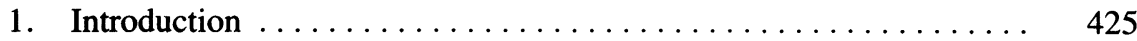

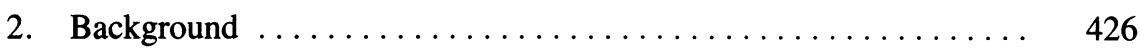

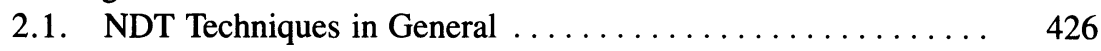

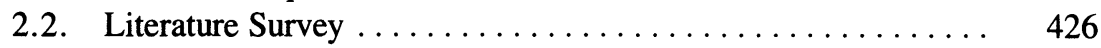

2.3. Physical Model Considerations $\ldots \ldots \ldots \ldots \ldots \ldots \ldots \ldots 428$

3. Ultrasonic Physics . . . . . . . . . . . . . . . . 432

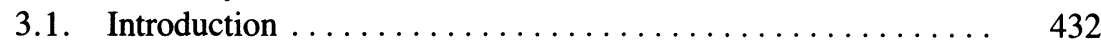

3.2. Ultrasonic Wave Generation and Wave Velocity ........ 432

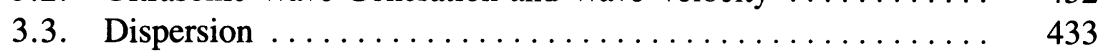

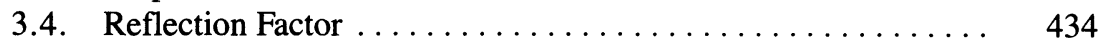

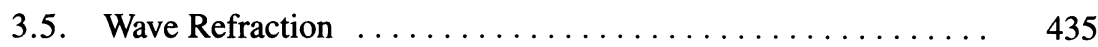

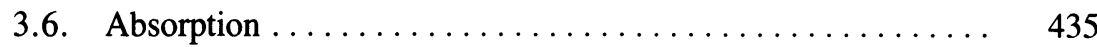

3.7. Ultrasonic Field Analysis $\ldots \ldots \ldots \ldots \ldots \ldots \ldots \ldots \ldots . \ldots \ldots$ 


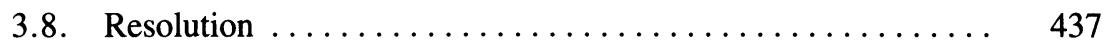

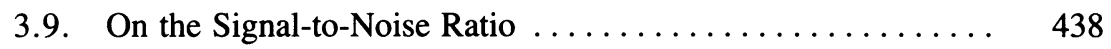

4. Feature-Based Systems and Advanced Analysis ........... 438

References .............................. 447

About the Contributors . . . . . . . . . . . . . . . . . . . 449

Author Index $\ldots \ldots \ldots \ldots \ldots \ldots \ldots \ldots \ldots \ldots \ldots \ldots \ldots \ldots \ldots \ldots$

Subject Index $\ldots \ldots \ldots \ldots \ldots \ldots \ldots \ldots \ldots \ldots \ldots \ldots \ldots \ldots$ 
Adhesive Bonding 